\title{
Adaptive Changes in the Psyche of Homo sapiens during the Period of the Singularity
}

\author{
Alexander G. Kruglov, PhD, ScD*; Andrey A. Kruglov, PhD \\ Central Research Institute of Radiation Diagnosis \\ Moscow, the Russian Federation
}

\begin{abstract}
The perception of environmental images that are not in causal relationships and are not interconnected sequences forms what is called clip thinking (ClipT). The concomitant decrease in the analytical-synthetic psychic functions is the result of "images" from the environment (not constructs of the psyche of the subject) being crowded out by a sequence of other images, and so are not kept in the field of voluntary attention for sufficient time for fixation. As a result, along with analytical and synthetic functions, from the sequential series of operational functions of conceptual thinking (ConceptT), come the closing steps of the perception phase - the default mode network (DMN) activity. The result is changes in the reciprocal relationship between the perception and processing of incoming information. The deficiency changes in DMN cause an imbalance of structures that provide "focus and defocus," difficulty fixing attention and filtering information, interruption of social relations, and other disruptions. ClipT forms the ability to perceive a larger (compared to ConceptT) amount of short volumes of information per unit of time, with an increase in the total number and range of heterogeneous units of incoming information, increasing the multitasking of thinking and allowing the simultaneous execution of several types of activities. The prospective dynamics in the development of ClipT forms global unified communicative codes with the potency of the functional association of subject groups that do not have stratification and quantitative restrictions. The distribution of information in these associations, which have almost identical characteristics of operational symbols (OSs), and the high speed of distribution of content on an unlimited scale of social networks, is similar to infection mechanisms during epidemics. ClipT is at the initial stages of development, adapting the psyche to accelerating changes in the environmental information parameters. We believe that ClipT and ConceptT are various interfering processes of the transforming psyche of Homo sapiens. The development of ClipT creates the conditions for the psyche to transition to a new qualitative level, with a change in structure and a transition to a new class of systems. In the conditions of increasing density, speed, randomness of the information flow and increasing degree of uncertainty, we consider the new phase transition as the attractor of the described changes in thinking (the psyche as a whole) of HS - the singularity period determining the changes (transformation of thinking is the most accessible integral research feature of significant, but less noticeable changes in the psyche as a whole) that take place with the future final state of a unified information system, including external devices and adaptive integral changes in the properties of the psyche as a whole. (International Journal of Biomedicine. 2020;10(2):95-100.)
\end{abstract}

Key Words: clip thinking $\bullet$ conceptual thinking $\bullet$ singularity $\bullet$ attractor $\bullet$ evolutionary development

\section{Abbreviations}

CC, creative construct; ClipT, clip thinking; ConceptT, conceptual thinking; DMN, the default mode network; HS, Homo sapiens; OS, operational symbol; PT, paralogical thinking; TC, thinking in complexes

\section{Basic Part}

The aim of the present work was to identify some vectors of the development of the human psyche by analyzing the interconnections of Concept T and ClipT during the period of evolutionary phase transition.

\section{Materials and Discussion}

We consider Concept T in the current period of time as a derivative of syllabic writing, having a standard order of signs: the alphabet (in contrast to the subject, pictographic, hieroglyphic, and other types of communication): i.e. as a 
variant of a textual civilizational cultural code that brought together oral and written speech. Throughout the history of the development of HS, the development and dissemination of "textual" ConceptT in various historical eras was limited to social groups where writing was predominantly utilitarian in nature (with exceptions that are not significant in the aspect under consideration). The total spread of writing and the concomitant development of the "text" ConceptT arose only with the beginning of printing (Gutenberg, 1439). The global spread of book printing has triggered the emergence of print ("textual") culture; the perception, processing, and dissemination of information; and the development of Concept T as the dominant form of thinking that forms the psyche as a whole.

We consider thinking as an integral function of the psyche, a special kind of mental and practical activity of HS, including the operational activity of a cognitive and transformative nature, which has several successive stages. In its most general form, this activity is visual-effective thinking (object-manipulative) at an early age; visual-figurative thinking, in which the subject thinks only with specific images (up to $\sim 7$ years of age); figurative thinking (verbal-logical), using concepts without having had specific experience of them ( $>12$ years of age). Operational functions of thinking are a) comparison - detecting similarities and differences between objects and phenomena; b) analysis - allocating essential features of objects; c) synthesis - combining the selected properties into a single whole; d) abstraction - allocating the main (from the point of view of the subject) signs separated from secondary signs; e) generalization - an ability to combine the main distinguished properties of an object into a single whole. "Concepts" are formed by the consistent dynamics of psychological changes (>12 years of age) ${ }^{(1,2)}$ which are the result of independent activity (operating with a word). All mental functions (associations, memory, attention, etc.) participate in this process, but do not create it. A "concept" is formed by a word acquiring a certain semantic meaning. ConceptT does not exist outside of speech-thinking, without words, in contrast to instincts and drives. The significatory meaning of a word, its use as a means of "concept" formation means a transition to operational activities, mediated by semantic meanings, hence to ConceptT. ConceptT forms the possibility of finding and highlighting significant features of an object, and qualifying and sorting those features into a homogeneous series of signs characterizing the object. ${ }^{(1,2)}$ The next stage is the sorting of objects, which allows one to combine in homogeneous groups not signs, but objects, forming an integral picture of the visible world and building a continuum of the environment as a whole.

The evolutionary bifurcation of hominids took place, presumably, as a result of the emergence of a new subsystem of the psyche- "creativity"-in the HS subspecies $(\sim 50,000$ years ago). ${ }^{(3)} \mathrm{We}$ understand creativity as a specific feature of the psyche of HS, the ability to produce prognostic hypotheses that cannot be derived directly from the initial conditions. As a result of the development of creativity, there appeared the ability to think symbolically, as well as to construct abstract images and adaptive forms of purposeful (non-reactive) behavior corresponding to the conditions and degree of uncertainty. The creative construct (CC), originally "image of the goal," was transformed into a fragment of the environment with the functions of a controlling object. ${ }^{(3)}$ The combination of images and meaningful meanings in $\mathrm{CC}$ created abstract thinking, the development of which led to the development of symbolic thinking. Being the final segment of the pyramid, consisting of representations, mythologies, myths, logical constructions, etc., the final product of thinking was formedthe "symbol" (Image+Sense), ${ }^{(4)}$ which has a finite number of signs, correlated with the amount of voluntary attention (consciousness) and perceived as an indivisible object. Each $\mathrm{CC}$, arising as a neural association that realizes an actualized need ${ }^{(3,5)}$ acquires the properties of a dominant focus with the corresponding equivalents. Dominant foci displaced from the "conscious" area constitute structured neural constellations that are in interferential relationships and are actualized by repeated invariant sensory patterns from the sensory organs or arsenal of needs (neural pathways). The totality of these functional neural constellations makes up the content of the "subconscious", where each structural component has the potential for "readiness" to motivate the subject during actualization. Creating a system of virtual representations and reducing the degree of uncertainty, the $\mathrm{CC}$ sequence structures and organizes the system of subject-object relations. Thus, in the phylogenesis of HS, a precedent has been noted for integrating a new psyche subsystem, creativity, with reformatting of the psyche as a whole and the emergence of qualitatively new operational functions (creation and dissociation of a symbol with behavioral equivalents). ${ }^{(3,5)}$

ClipT is formed by the perception of environmental images that are not in causal relationships, as well as by those that are not interrelated sequences but being in sufficient time in this environment. ${ }^{(6,7)}$ A 6-fold excess of the efficiency of presenting the sequence of the imagery (suggesting a readymade solution) over the text (with sequential conceptual processing and the final conclusion) is established. A decrease in the level of analysis and synthesis of information obtained in the form of a finished image associated with ClipT is a consequence of the fact that the "image," not being a construct of the psyche of the subject and not holding in the field of voluntary attention (consciousness) long enough for fixing (and subsequent processing), is crowded out by a sequence of other images, often outside of logical and sequential relationships. We believe that the sequential series of operational functions of thinking changes, which are the closing steps of the perception phase (the DMN activity) in ConceptT. Thus, the reciprocal relationships between the brain's systems for perceiving and processing incoming information are changes, and their imbalance arises. In connection with a deficiency of changes in DMN, the balance of the joint work of structures that ensure "focus" and "defocus" is upset. ${ }^{(8)}$ The results are a) difficulty in fixing attention; b) disturbances of filtering (necessary/ unnecessary) information; c) an easy distraction from the main current task; d) a decreased flexibility of thinking; $\left.{ }^{(9,10)} \mathrm{e}\right)$ disruption of the chain of "social ties". ${ }^{(1,12)}$ The time lag of a sequence of ConceptT is much longer than ClipT. Possessing an advantage in the speed and volume of perceived fragmented 
information, ClipT loses in the perception and processing of a long linear sequence, possibly playing the role of a "filter" in the conditions of information overload and randomness of the growing information flow. ${ }^{(13)}$ In other words, ClipT forms the ability to perceive a larger (compared to Concept $\mathrm{T}$ ) amount of short volumes of information per unit of time, with an increase in the total number and range of heterogeneous units of incoming information, increasing the multitasking of thinking and allowing the simultaneous execution of several types of activities. ${ }^{(14)}$

We note, in solidarity with the "theory of incompleteness," ${ }^{(15)}$ that any depth of penetration into the studied object, even for the most developed ConceptT, is always relative, reaching the limits possible for a given subject, being only a "striving" in this direction, with limited achievement. In other words, the productive results of ConceptT, despite the obvious effectiveness in all areas of human activity, are superficial with respect to the structure of any object of study (limited by the current level of knowledge). We believe that a comparison of the effectiveness and productivity of ConceptT and ClipT is premature, since important factors are still not sufficiently known - the development vectors and the distant characteristics of ClipT in the initial phases of development, the proportionality limits of relationships with external devices (forming a new configuration of both personality and society), and the long-term results of the practical, operational effectiveness of ClipT in the face of accelerating changes in the information and social environments.

Many reasons are advanced for the formation of ClipT in modern man. We consider, without considering it sufficiently complete, one of the classifications: ${ }^{(16)}$ the development of modern technologies, which are accompanied by a significant increase in the information flow; 2) the need to receive a large amount of information more quickly; 3) multitasking and expanding the range of information flow; 4) the accelerating rhythm of life and increased diversity of simultaneously arising tasks; and 5) the growth of dialogicity at different levels of the social system. ClipT differs from Concept $\mathrm{T}$ in that what is absent in ClipT is context-i.e. semantic completeness, ${ }^{(17)}$ the meaning of the components of the text (speech), and the semantic connections of fragments of the context; and as a result, ClipT lacks analytical and synthetic functions with the subsequent difficulty in conjugating the perceived fragments into a generalized picture. A fragmented (without internal semantic connections) mosaic of perceived images, compared with each other, according to an algorithm unknown to us, forms ideas about the environment, which may not correspond to reality, ideas about which (i.e. reality) are formed with the participation of ConceptT. We believe that the subjective interpretation of the reflection of environmental phenomena (i.e. "experience," (18) which is formed with the participation of ClipT) constructs ideas about the environment that are different from those created through ConceptT, on the basis of which new forms of adaptive behavior are formed that are adequate to the current ideas (different from those that have become standard). In other words, the gradients of personality development and the formation of adaptive (including social) behavior under the influence of ConceptT and ClipT, having differences in content and speed (with a predominance of ClipT) characteristics, also have diverging development paths.

The transformation of modern society into "electronic, informational," through electronic means of communication, forming ClipT, partially returns human thinking to the pretext era, with the exhaustion of the role of a linear sequence of characters as a basis for cultural development. ${ }^{(19)}$ For a quantitatively growing part of the population that has undergone informational transformation, which will become the general population of the future, what is fundamentally new is the constructive formation of perception and modeling of the image of the environment, based on the perception of information segments ("slicing"), as the preferred method of perception. A preference for perception is given to multiple incoherent episodes, pieces of information, in contrast to the completed plot media models..$^{(20,21)}$

Given the growing global homogeneity (informative and figurative) and the similarity of information codes that form and format ClipT (unification of degrading informational occasions, informative and figurative media products, the unifying impact of social networks and entertainment, memes, etc.), we consider the following conclusion to be logical. Thus, in contrast to Concept $\mathrm{T}$, the development gradient and the main vector of perspective dynamics of ClipT leads to the achievement of a finite volume of unified OSs, the narrowing range of which will cover significant numbers of subjects (possibly populations), regardless of geographical, linguistic and other differences. In other words, global unified communication codes are gradually being formed, creating network associations that are not scalable and are organized by horizontal connections. Features of ClipT (reduction of analytical and synthetic functions, unification of OSs, etc.) form a functional union of a "homogeneous series" of subjective unstratified associations that contain an identical cultural communication code, where the mechanism of information dissemination (proximity of meanings and OSs with reduced filtering functions, high speed of content distribution on an unlimited scale of social networks, etc.) is close to that of infection mechanisms during epidemics.

Discrete signs of pre-book (pre-text) style in cultural communication, forming ClipT, arose long before the advent of computer games and social networks. ${ }^{(22)}$ Functionally, this style is the perception of the connections of one's own perceptions with equivalent connections of the objects of perception, i.e. partial identification of oneself with the object of perception. In ontogenesis, this is the phase of adolescent "thinking in complexes" (TC), where complexes are not concepts, but their functional equivalents. TC is a functional precursor of ConceptT, structurally included in the design of ClipT. In TC, each item entering a complex does not merge with another one, retaining its identity (for example, ancient languages: Chinese, Latin, etc.). In this phase, any connection can arbitrarily group actual (non-abstract, non-logical) objects. That is, in contrast to Concept T, the basis of which is a single significant connection (including abstract, logical); in the TC, regardless of the type of complex, the basis for combining objects is actual (random) communication. TC is diffuse, and does not have certain outlines and boundaries. The form 
of TC is ambivalent, representing: a) generalization, but different in structure from the concept; b) an image, «mental drawing of the concept.» ${ }^{(2)}$ The result of TC is a difference in the values that define different objects with the same word. In other words, each specific object entering the complex retains its identity, not combining with others. The result is a participation (relationship) that primitive thought establishes between different objects, considered either as partially identical, or as influencing each other, but in reality having neither spatial contact nor any other causal connection. ${ }^{(2,23)}$ At the basis of the etymological definitions of a "word." a very significant part did not have logic or connections established in "concepts." That part was replaced by figurative complexes with the allocation of a random attribute that determined the name of the object.

Without bringing all phases of the formation of ConceptT in ontogenesis, we note the main, final stage: the ability to synthesize abstracted signs, where the word (sign) is the basis for distinguishing the sign and the subsequent synthesis of signs into a higher operational function — a symbol (Image+Sense). ClipT, surpassing Concept T in speed and range characteristics, while being deficient in the depth of analysis, synthesis, and filtering of values, differs in the final result - the OS. The OS of ClipT is different from the OS of ConceptT. With the identical initial information field, the semantic content of the OS changes. OSs of ConceptT and ClipT, preserving the identity of the "image," have substantial (semantic) differences, as a result of differences in principles of constructing fragments of the information field in the OS during ClipT. An OS with ClipT has a superior (in comparison with Concept $\mathrm{T}$ ) range of perceived objects and a large information capacity with less analytical and synthetic generalization. Insufficient conceptual, analytical and synthetic depth at a high processing speed, suggests the fragmentation of the final informational figurative resource with arbitrary plot integration into a single whole. The intra-plot connections in ClipT create the conditions for the formation of logical circuits that combine figurative series in way according to an algorithm unknown to us. ClipT arises, in our opinion, as an adaptive version of the development of the psyche with a rapid chaotic increase in the quantitative characteristics and variability of the choice of stimuli from the information field, as well as with many alternatives and the absence of clear criteria for optimality and efficiency, increasing the degree of uncertainty of the system as a whole. We note the existence of the concept of uncertainty as a fundamental property of nature. ${ }^{(24)}$ The general tendency for the development of ClipT at the present stage, according to formal features, can be defined as the archaization of thinking. Constructively, ClipT includes significant fragments of TC and PT, ${ }^{(25)}$ including the possibility of identifying the subject "I" with an external object by abstraction and "transfer" (imagination, empathy) of personal properties to an external (external) object, i.e. splitting the representation of the integral personality. ${ }^{(26)}$

We believe that we describe the development of a new type of thinking of HS, conventionally called ClipT, that has arisen and is being formed in the general cultural environment with the participation and on the basis of ConceptT, in conditions of rapid growth and increasing randomness of the information flow. HS at the next stage of technological development, in the dynamics of the rapid development of the electronic digital environment, received external devices that provide inexhaustible memory resources, computational speed, and synthesis of previously incomparable objects, creating a virtual environment with a migration of a decision center, the possibility of dialogue (with an opponent whose logic, memory and speed of thinking are qualitatively and quantitatively different from the subjective decisions), and other characteristics. Interaction with an external device has created the possibility of relationships that can be partially defined as "interpersonal," which already leads to depletion (crowding out) of social ties and a change in the whole configuration and parameters of the social spectrum of the external environment. In other words, the dynamics of the development of ClipT (with the gradual displacement of ConceptT) indicates the HS psyche is adapting to the conditions of an increasing information flow, its randomness, and a gradual change in the ratio of roles in a pair: operatordevice. We intentionally do not give quantitative values for the increase in density, speed and quality of the information flow, taking them as a fact confirmed by many studies. Our goal is high-quality adaptive changes in the psyche of HS in the conditions of the information environment with a rapid change in parameters.

ClipT is at the initial stages of formation, being a measurable indicator of the adaptation of the HS psyche to accelerating changes in the basic information parameters of the environment. We believe that we do not describe the transformation of ConceptT into ClipT; these are two different, partially interfering processes of the psyche that adapts to significant and accelerating changes in the environment. Considering the psyche as an active, distributed system consisting of functional subsystems, we believe that the emergence of a new integrating subsystem, ClipT, creates (partially crowding out Concept T) conditions for the transition to a new qualitative level of the system as a whole, with a change in the structure of functional properties and transition to a new class of systems.

The theory of evolution of the biosphere and society is formulated as a synergistic model of development, ${ }^{(27-29)}$ where resource and technological-humanitarian crises, as well as environmental degradation, can be productive (producing phase transitions). One of the decisive factors for survival during phase crises is the excessive internal diversity of the system. That is, at the onset of the evolutionary crisis, species forms that did not have evolutionary advantages before found a deterministic equivalent response, an adaptive response to the crisis, which made them a new system-forming factor; ${ }^{(28)}$ one of the signs of such an adaptive reaction, we believe the formation of ClipT.

Modification of HS, through the emergence and development of new mental functions (creativity, symbolic thinking, variable adaptive behavior that initiated technological development), which were phylogenetically transformed into a significant and then leading factor in evolution. ${ }^{(3,5)}$ In our earlier data (including graphs and velocity characteristics of 
evolutionary phase transitions), ${ }^{(5)}$ we considered the effects of the attractor on the evolutionary development of HS. We understand the attractor as the final area of the inevitable convergence of the phase trajectories of a complex system, the attraction of which draws into it the many possible states of the trajectories of the systems, determined by different initial conditions. In other words, the final state has not yet been achieved, but it is already having an effect, like the goal chosen by the system. The dynamics of the accelerating phase transitions (decreasing over time intervals) of HS evolution is defined as a sequence that has a limit (singularity of evolution). ${ }^{(30,31)}$ Since the "singularity point" is a mathematical abstraction, it is proposed to consider it not a "point," but a "period of singularity."(32)

We believe that the singularity of evolution, as an attractor, affects the changes in the HS psyche, including the formation of changes in thinking - the most obvious, accessible for perception and study (in large samples), is an integrated set of signs of changes in the psyche as a whole for a historically short (observable) period of time. We consider the complex of the described changes in thinking, deviations from the standards of Concept T, accompanied by changes in the structure, content and basic vectors of the development of thinking (conventionally called ClipT), as a developing, adaptive, systemic change in the HS psyche as a whole. These systemic changes in the psyche are in the process of adapting to the development vectors of the singularity, with subsequent transformation into a single information system, including external devices and concomitant changes in the personality's integral properties. That is why we do not support the alarmist conclusions of some psychologists about the transformation of thinking as presumably degrading; rather, we believe that the psyche is at one of the initial stages of adaptation to the information environment that is developing at an accelerating pace.

\section{Conclusion}

Thinking, as an integral function of the psyche, is currently being transformed under conditions of a significant increase in the density, speed, and randomness of the information flow. On the basis of the dominant ConceptT, a new type of thinking is being formed, conditionally called ClipT, which adapts the psyche to informational changes in the environment. ClipT emerged as a result of the increasing speed and randomness of the information flow, which in turn led to the perception of environmental images that are not in causal relationships. The formation of a new type of thinking with higher speed characteristics and other principles of the formation of structural elements, and changes in the construction of the semantic part of the final product ("symbol"), is accompanied by changes (possibly partial loss) in the closing steps of the perception phase, the DMN activity, which leads to a contravention in the reciprocal connection of the perceiving and processing information systems of the brain. OSs of ConceptT and ClipT, while preserving the identity of the image, have substantial semantic differences, as a result of the construction (according to an algorithm unknown to us) of fragments of the OS information field in
ClipT. Gradients of the development of the psyche and forms of adaptive behavior under the influence of ConceptT and ClipT have differences in content and speed characteristics, as well as diverging development trajectories. We believe that we are not observing the transformation of ConceptT into ClipT, but two different, partially interfering processes of the HS psyche, which adapts to accelerating changes in the information environment. The integration of a new subsystem (ClipT) creates the conditions for the transition of the psyche to a new qualitative level, with a change in functional properties and a transition to a new class of systems. In the conditions of increasing density, speed, randomness of the information flow and increasing degree of uncertainty, we consider the new phase transition as the attractor of the described changes in thinking (the psyche as a whole) of HS - the singularity period determining the changes (transformation of thinking is the most accessible integral research feature of significant, but less noticeable changes in the psyche as a whole) that take place with the future final state of a unified information system, including external devices and adaptive integral changes in the properties of the psyche as a whole.

\section{Competing Interests} interests.

\section{References}

1. Vygotsky LS. Thinking and Speech (1934). Natsionalnoe Obrazovanie; 2019:368 pp. [In Russian].

2. Vygotsky LS. Selected psychological works. M. Academic science of the RSFSR; 1956:519 pp. [In Russian].

3. Kruglov AG. Creativity as a Determinant of the Development of Homo Sapiens. International Journal of biomedicine. 2016;6(4):298-302.

4. Losev AF. Essays on ancient symbolism and mythology. M. Misl.;1993:962 pp. [In Russian].

5. Kruglov AG. Mental Activity as Attractor of Evolutionary Development of Homo sapiens. International Journal of biomedicine.2017;7(4):330-334.

6. Girenok FI. Clip consciousness. M. Akademicheskii Prospect; 2014: 249 pp. [In Russian].

7. Girenok FI. Clip consciousness. M. Prospect;2016:256 pp. [In Russian].

8. Anticevic A, Cole MW, Murray JD, Corlett PR, Wang XJ, Krystal JH. The role of default network deactivation in cognition and disease. Trends Cogn Sci. 2012 Dec;16(12):58492. doi: 10.1016/j.tics.2012.10.008.

9. Vatansever D, Manktelow AE, Sahakian BJ, Menon DK, Stamatakis EA. Cognitive Flexibility: A Default Network and Basal Ganglia Connectivity Perspective. Brain Connect. 2016 Apr;6(3):201-7. doi: 10.1089/brain.2015.0388.

10. Sali AW, Courtney SM, Yantis S. Spontaneous Fluctuations in the Flexible Control of Covert Attention. J Neurosci. 2016 Jan 13;36(2):445-54. doi: 10.1523/JNEUROSCI.2323-15.2016.

*Corresponding author: Alexander G. Kruglov, PhD, ScD. Central Research Institute of Radiation Diagnosis. Moscow, the Russian Federation.E-mail: krag48@mail.ru 
11. Li W, Mai X, Liu C. The default mode network and social understanding of others: what do brain connectivity studies tell us. Front Hum Neurosci. 2014;8:74. eCollection 2014.

12. Mars RB, Neubert FX, Noonan MP, Sallet J, Toni I, Rushworth MF. On the relationship between the «default mode network» and the «social brain». Front Hum Neurosci. 2012 Jun 21;6:189. doi: 10.3389/fnhum.2012.00189. eCollection 2012.

13. Klyuchnikov SJ. Master of life: psychological defense in society. M. Belovodye; 2001:592 pp. [In Russian].

14. Rosen L. Me, My Space and I: Parenting the Net Generation. NY.; 2007: 258 pp.

15. Gödel K. Some Basic Theorems on the Foundations of Mathematics and Their Implications', in (Gödel 1995), pp. 304-323. 16. Frumkin KG. Clip thinking and the fate of linear text. Electronic resource. Topos: literary and philosophical journal. 2010;9. Available at: http://www.topos.ru/article/7371. [Article in Russian].

17. Golovin SJ. Dictionary of practical psychologist. Mn.: Harvest;1998:201 pp. [In Russian].

18. Rogers C, Freiberg H. Freedom to Learn. M. Smysl (Translation from English into Russian); 2019:527 pp.

19. McLuhan M. The Gutenberg Galaxy: The Making of Typographic Man. M. Academic project (Translation from English into Russian); 2005:496 pp.

20. Toffler E. Shock of the future. M. AST; 2008;560 pp. [In Russian].

21. Toffler E. The Third Wave. M. AST; 2010:784 pp. [In Russian].

22. Bukatov VM. [Clip changes in the perception, understanding and thinking of modern schoolchildren are an annoying new formation of the «post-industrial structure» or the long-awaited resuscitation of the psychic nature]. Aktualnye Problemy Psikhologicheskogo Znaniya. 2018;4(49):5-19. [Article in Russian].

23. Piaget J. The Psychology of Intelligence. SPB. (Translation from English into Russian); 2003:192 pp.

24. Kravchenko AO. [Abstracts on uncertainty]. Nauka i Prosveschchenie. 2014;4:14-19. [Article in Russian].

25. Levy-Bruhl L. citation Enikaev MI: Psychological Encyclopedic Dictionary. M.;2010: 560 pp. [in Russian].

26. Philosophy: Encyclopedic Dictionary. Ed. AA Ivin. M.: Gardariki; 2004:1072 pp. [in Russian].

27. Nazaretyan AP. Civilization crises in the context of Universal History (synergetics-psychology-forecasting). M. Mir;2004:368 pp. [in Russian].

28. Panov AD. Universal evolution and the problem of the search for extraterrestrial intelligence (SETI). M.LKI; 2008:208 pp. [in Russian].

29. Snooks GD. The dynamic society. Exploring the source of global change. London, NY, Routledge; 1996:491 pp.

30. Panov AD. Singular point of history. Social sciences and modernity. M. MSU;2005:122-137. [in Russian].

31. Kapitza SP. [The phenomenological theory of word population growth]. Uspekhi Fizicheskikh Nauk. 1996;166(1): 63-80.[Article in Russian].

32. Konstantinov AI. Singularity of planetary evolution: further refinement of the model. Evolution (Megahistory and global evolution). Volgograd; 2015:58-64. [in Russian]. 